\title{
STABILITY OF ANNULAR FLOW AND SLUGGING
}

\author{
D. D. JOSEPH, A. C. BANNWART and Y. J. LIU * \\ Dept. of Aerospace Engineering and Mechanics \\ University of Minnesota \\ 107 Akerman Hall, 110 Union St. SE, Minneapolis, MN 55455, USA
}

(Revised on May 30, 1996)

\begin{abstract}
In this work we propose an effective viscosity criterion for the stabilization of annular gas-liquid and liquid-particle flows and an inertial mechanism which drives waves into slugs in slugging gas-liquid flows. Annular flow is stable when the fluid having the higher effective viscosity occupies the core region and the lower viscosity fluid is the annulus. The eddy viscosity criterion is shown to be very consistent with published work on annular flow transitions in horizontal and vertical gas-liquid flows. It also applies to a variety of liquid-solid and gas-solid flows. In the second part of the paper we propose a mechanism for explaining the growth of initially small waves and initiation of slugs in gas-liquid flow.
\end{abstract}

Key Words: annular gas-liquid flow, stability criterion, transition, slugging, core flow.

\section{Phase arrangement and stability of annular flows}

It is well known that, in liquid-liquid flow or gas(vapor)-liquid flow in a pipe, a core-annular flow with one fluid in the core and the other fluid in the annulus appears under certain flow conditions. The oil forms the core in an oil-water core annular flow, and the gas phase is always in the core in a gas-liquid annular flow.

Most widely accepted theories for horizontal gas-liquid flow postulate that transition to annular flow takes place when there is not too much liquid (the liquid level is below pipe center line) and the conditions are such that a finite amplitude wave on the stratified surface will grow [Taitel and Dukler, 1976, hereafter TD]. For vertical upward flow the transition to annular flow is said to occur when the gas velocity reaches the minimum necessary to suspend a liquid drop of critical size [Taitel, Bornea and Dukler, 1980, hereafter TBD].

The stability of annular flow can be discussed without any consideration of the particular mechanism by which the transition starts. It seems to be a rather general, if not universal principle that the less viscous fluid migrates to the region of high shear, lubricating the flow (see, for example Joseph and Renardy [1993] or Merkle and Deutsch [1990]). The same principle may also govern other two-phase flows when an effective eddy viscosity (molecular viscosity plus turbulent viscosity) is used. Here we argue that core flows with a less viscous fluid inside can be stable in turbulent flow when the eddy viscosity in the core is larger than in the annulus. Stable annular flow appears only when the gas flow in the core is very highly turbulent with a higher effective viscosity than the liquid in the annulus.

Annular gas-liquid flow is regarded as a forced convection flow in which there is a liquid film on the wall with a continuous gas core in the center of the channel. The simplest picture of this flow pattern is to assume a laminar regime for the thin liquid film and a fully turbulent regime with no entrained droplets for the gas core. Neglecting the molecular viscosity of the gas, we have:

$$
\begin{gathered}
\mu_{e f f, L}=\mu_{L}, \\
\mu_{e f f, G}=\mu_{T, G}
\end{gathered}
$$

*present address: 3M Corporation, 3M Center, 236-2S-12, St. Paul, MN 55144. 
where $\mu_{L}$ is the molecular viscosity of the liquid and $\mu_{T, G}$ is the turbulent viscosity of the gas flow. A conservative estimate of the eddy viscosity of the gas can be obtained from the well-known flat plate results neglecting any influence of the typically wavy interface in evaluating $\mu_{T, G}$. The turbulent velocity profile is very flat; thus the eddy viscosity of the gas rapidly reaches its value in the outer layer. This is given by the wellknown Clauser's formula [White, 1991]:

$$
\mu_{T, G}=0.016 \rho_{G}\left(U_{G}-U_{i}\right) \delta_{G}^{*}
$$

where $\rho_{G}$ is the density of the gas, $U_{G}$ is the average velocity of the gas, $U_{i}$ is the velocity at the interface and $\delta_{G}^{*}$ is the displacement thickness of the gas flow. By assuming a one-seventh power law for the gas and that $U_{G}>>U_{i}$ we can write

$$
U_{G} \delta_{G}^{*} \cong \frac{U_{G} D}{16} \cong \frac{J_{G} D}{16}
$$

where $J_{G}$ is the superficial velocity of the gas and $D$ is the tube diameter. The condition at which $\mu_{\text {eff, },}=\mu_{\text {eff }, L}$ can thus be stated as

$$
\frac{\rho_{G} J_{G} D}{\mu_{L}} \cong 1000
$$

Equation (5) holds when the liquid flow in the annulus is laminar. For example, consider an air-water flow at 1 bar and $25^{\circ} \mathrm{C}$ inside a $25 \mathrm{~mm}$ tube; equation (5) gives $J_{G}=30 \mathrm{~m} / \mathrm{s}$. This criterion is represented by the vertical line A on TD's flow map in figure 1. Almost the entire annular flow region corresponds to the condition $\mu_{e f f, G}>\mu_{e f f, L}$, which is possibly a significant result in view of the simplicity of the proposed model.

At high liquid flowrates the flow in the annulus becomes fully turbulent and equation (1) is re-written as

$$
\mu_{e f f, L}=\mu_{T, L}
$$

The turbulent viscosity of the liquid flow $\mu_{T, L}$ is modeled the same way as the gas flow leading to the expression

$$
\mu_{T, L}=0.016 \rho_{L} U_{L, \delta} \delta_{L}^{*}
$$

where $\rho_{L}$ is density of the liquid, $U_{L, \delta}$ is the average velocity of the liquid film and $\delta_{L}^{*}$ its displacement thickness. Assuming once more a one-seventh power law for the velocity profile in the film, we get

$$
U_{L, \delta} \delta_{L}^{*}=U_{L, \delta} \frac{\delta}{8}=\frac{J_{L, \delta} D}{32}
$$


Figure 1. Taitel and Dukler's [1976] flow map of horizontal air-water flow at 1 bar and $25{ }^{\circ} \mathrm{C}$. The pipe diameter is $25 \mathrm{~mm}$. The solid lines stand for the theoretical transitions proposed by Taitel and Dukler. The slashed lines represent the experimental results given in Mandhane, Gregory and Aziz [1974]. $\mu_{\text {eff }, G}=\mu_{\text {eff }, L}$ on the dashed-dot line.

where $\delta$ is the thickness of the liquid film and $J_{L, \delta}$ its superficial velocity. Thus for turbulent film flow the condition $\mu_{e f f, G}=\mu_{e f f, L}$ can be written as

$$
\frac{\rho_{G} J_{G} D}{\mu_{L}}=\frac{1}{2} \frac{\rho_{L} J_{L, \delta} D}{\mu_{L}}
$$

Equation (9) holds for fully turbulent film flow. The intercept between equations (9) and (5) is obtained as

$$
\frac{\rho_{L} J_{L, \delta} D}{\mu_{L}}=2000
$$

which is the expected result. Since the liquid flowrate is high, the amplitude of interfacial waves grows dramatically and bridging of the cross section is incipient. Thus a considerable amount of liquid actually flows in the core and $J_{L, \delta}$ is much smaller than $J_{L}$. If $C$ is the fraction of the liquid flowrate that flows in the core then 


$$
J_{L, \delta}=(1-C) J_{L}
$$

Assuming now a constant value for $C$, equations (9) and (11) can be represented by straight lines $C=0.90$ and $C=0.97$ which are actually parallel to TD's flow boundary $\mathrm{B}$ in figure 1.

The condition at which $\mu_{e f f, G}=\mu_{e f f, L}$ can thus be summarized as follows:

$$
\frac{\rho_{G} J_{G} D}{\mu_{L}}=\left\{\begin{array}{cccc}
1000 & \text { if } & \frac{\rho_{L} J_{L, \delta} D}{\mu_{L}} \leq 2000 & \text { (boundary } A \text { ) } \\
\frac{1}{2} \frac{\rho_{L} J_{L, \delta} D}{\mu_{L}} & \text { if } & \frac{\rho_{L} J_{L, \delta} D}{\mu_{L}}>2000 & \text { (boundary } B \text { ) }
\end{array}\right.
$$

where $J_{L, \delta}$ is given by equation (11). The value $C \cong 0.95$ is suggested if the boundary (B) for turbulent film flow is to be matched with TD's boundary.

A comparison of equation (12) with existing maps on the transition to annular flow for upward gas-liquid flow in vertical tubes is shown in figure 2, which was extracted from TBD's work. This figure refers to air-water flows at low pressure and tube diameters ranging from 2 to $6 \mathrm{~cm}$. Thus it can be used to discuss the effect of the tube diameter on the transition. The shaded area represents the experimental data published by different authors (the boundary proposed by Griffith and Wallis [1961] gives too low transition superficial gas velocities and has not been considered here).

It is well known that TBD's transition model shows no effect of the tube diameter. However, as it can be seen in figure 2, even for low liquid flowrates there is a wide discrepancy in the data, because different authors may use different criteria to qualify visually the onset of the transition. Due to such subjectivity it is not possible to conclude on the effect of tube diameter on the basis of available experimental data. In our criterion, the stabilization of annular flows involves the inertia of the gas in the tube, so the effect of the diameter appears naturally in the vertical boundary A.

As can be observed in figure 2, TBD's model does not exhibit an inclined part (boundary B) at high liquid flowrates. This feature is displayed by our model and seems to be confirmed by others investigators (as for example Duns and Ros [1963]). Notice that this boundary does not depend on tube diameter.

According to TBD's model the transition to annular flow is not affected by liquid viscosity which appears in our stability criterion. Of course, stability and transition are different. However, we think that TBD's transition criterion might fail if the liquid phase is highly viscous. In this case, even though the gas stream may have a minimum velocity necessary to lift a small droplet (as in TBD's model), it might not be able to displace the liquid towards the wall. This may require much more inertia for the gas. Our model gives precisely the minimum amount of inertia necessary for this task.

Ordinary lubrication layers occur frequently in gas-solid and liquid-solid twophase flows, for example, in the self-lubricating flows of suspensions, a thin fluid layer appears on the wall (for example, see Persello et al [1994]). In all these cases the core of suspension has higher viscosity than the liquid layer on the wall.

It is of interest to consider not so ordinary cases of lubrication layers in gas-solid and liquid-solid flows in which the fluid in the core aquires a huge eddy viscosity. This effect is well known in turbulent fluidized beds used in coal combustion in which the solids accumulate at the wall. 
Figure 2. Transition to annular flow for upward air-water flow at 1 bar and $25{ }^{\circ} \mathrm{C}$ inside vertical tubes with diameter ranging from 2 to $6 \mathrm{~cm}$. The solid line gives teh criterion for the theoretical transition proposed by Taitel, Barnea and Dukler [1980]. The shaded area indicates experimental data. The condition $\mu_{e f f, G}=\mu_{e f f, L}$ as given by equation (12) for $\mathrm{D}=2 \mathrm{~cm}$ and $\mathrm{D}=6 \mathrm{~cm}$ is represented by the dashed-dot lines.

An interesting theoretical study of the turbulent gas-solid upward flow in a fluidized catalyst cracker presented by Iske et al [1995] gives rise to segregation with particles on the gas in the center. Equation (9) of their paper defines an effective viscosity of the suspension as a function of the solid particle fraction:

$$
\mu_{\text {eff }, S}=\rho_{s} \xi(\varepsilon) \sqrt{\theta(\varepsilon)} f_{1}(\varepsilon)
$$

where $\rho_{s}$ is the density of the particle, $\varepsilon$ is the solid fraction, $\xi(\varepsilon)$ is the mean free path length of the particle in a suspension, $\theta=\frac{1}{3} \vec{u}^{\prime} \vec{u}^{\prime}$ is the pseudo-energy, and $f_{1}(\varepsilon)$ is a transport property of the particle phase. The functions $\xi(\varepsilon), \theta(\varepsilon)$ and $f_{1}(\varepsilon)$ obtained from collision theory of gas-solid suspensions can be expressed as (Sinclair and Jackson [1989]).

$$
\begin{gathered}
\xi(\varepsilon) \propto \varepsilon^{-1} \\
\theta(\varepsilon) \propto\left[\varepsilon\left(1+4 \varepsilon g_{o}\right)\right]^{-1},
\end{gathered}
$$




$$
\begin{gathered}
f_{1}(\varepsilon) \propto \varepsilon\left[\frac{\left(1+\frac{8}{5} \varepsilon g_{o}\right)^{2}}{g_{o}}+\frac{768 \varepsilon^{2} g_{o}}{25 \pi}\right], \\
g_{o}(\varepsilon)=\left[1-\left(\frac{\varepsilon}{0.6436}\right)^{1 / 3}\right]^{-1} .
\end{gathered}
$$

A plot of the effective viscosity function using the above equations in terms of the solid fraction is shown in figure 3 .

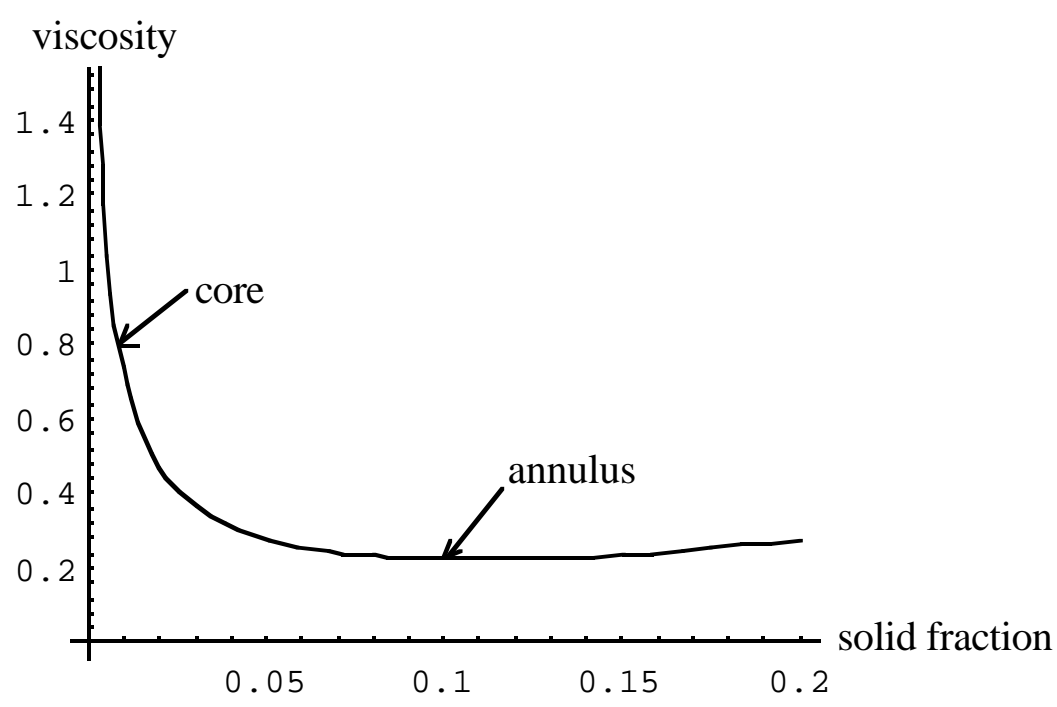

Figure 3. Effective viscosity function in a gas-solid suspension

The computation made by Iske et al shows a core region with very low solid fraction and a dense region near the wall (the annulus). We then conclude from their data that the effective viscosity is much higher in the core than in the annulus. Notice that the turbulence of the gas flow in the center has been neglected in the equations. If it is considered, the effective mixture viscosity in the core may be even higher.

Our proposition then is that the high viscosity material of a two-phase flow tends to migrate to the core where the shears are low and that an effectively high viscosity core can be created dynamically by turbulent fluctuations. The region near the wall where the shears are higher has a comparatively low viscosity.

\section{Slugging in gas-liquid flow}

This is apparently a different subject than the stability of annular gas-liquid flow. But the slugging mechanism to be proposed here is also motivated by core-flow of heavy oil; the mechanism which steepens the waves at the front of crest ought also to work in gas-liquid flow.

Various mechanisms for the initiation of slugs in gas-liquid flow have been proposed (see Fan, Lusseyran and Hanratty [1993]). However, the problem remains open. Here, we propose that the slugs are initiated by a nonlinear wave steepening 
mechanism associated with the high stagnation pressure at the front of wave crest on the liquid over which high speed gas passes. The formation of steep wave fronts in coreannular flows of heavy oils was proposed by Joseph in a paper on capsule transport in pipelines by Feng, Huang and Joseph [1995]. The wave distortion mechanism is robust and occurs both in low and high speed flows, in linear and nonlinear flow. In high speed flow over a body, without walls, on an unbounded domain, high stagnation pressures are at the front of the body, with pressure relief due to separation at the back. In lubrication theory even at low speeds, in Stokes flow, the pressure is high where the flow channel converges and low where it diverges. Such converging and diverging channels are formed by any wave near a wall with convergence at the front of a wave crest, divergence at the back. Naturally, a symmetric wave is unstable in such an environment, since the high pressure at the front will inevitably steepen the wave there and the low pressure at the back side will have the opposite effect. Secondary motions driven by high stagnation pressures are generated at steep wave fronts and the stagnation pressures grow strongly with an increase of the slip velocity. All these effects can be observed routinely in experiments and the effects of inertia in wave steepening have been obtained by direct numerical simulation by Bai, Kelkar and Joseph [1996] and are discussed in a review paper by Joseph et al [1997].

We are proposing that this dynamics which leads to large waves, steep at the front and smooth at the rear, as in figure 4 (b), applies even more strongly in high speed gasliquid flows where wave distortion due to high and low pressures is enhanced by the fact the mobility of water-like liquids is so much greater thant the mobility of heavy oils. The steep waves are definitely not long, but they are not so short as the ripples and roll waves which form on them by mechanisms related to Kelvin-Helmholtz instability.

The formation of steep asymmetric waves dos not always lead to slugging in core flows of heavy oil in water, so that the cartoon leading from steep waves in figure 4 (b) to slugs in 4 (c) is less universal than the one from 4 (a) to 4 (b). First, we note that in high speed gas flows the low pressures due to Bernoulli effects at wave crests would tend to pull the mobile water to the wall. If the wall were hydrophillic, these wave crests would stick to the wall entrapping gas to form slugs. An interesting question is if there is a difference in the dynamics of slugging with hydrophillic and hydrophobic walls.

A similar mechanism may also work in the case of breakdown of disturbance waves along a vertical gas-liquid interface. Hewitt and Hall-Taylor [1970] showed pictures (see figure 5) of breakdown of disturbance wave by "undercutting" observed in experiment by Lane [1951] for the shatter of droplets in high velocity air streams. Their explanation describes how the breakdown process develops but not how this "undercut" gets started. In fact, the stagnation pressure acting on the front of the crests of the small waves would tend to deepen troughs at the front and smooth them at the back, as in the cartoon shown in figure 5. Bernoulli effects should also be very important in a flow as fast as Lane's, pulling the wave out as shown in the cartoon. The "undercut" then follows subsequently. Of course, gravity is an actor in all this and the relative importance of the different effects is as yet unknown. 


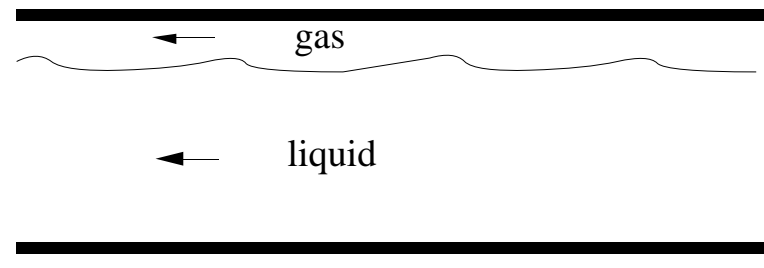

(a)

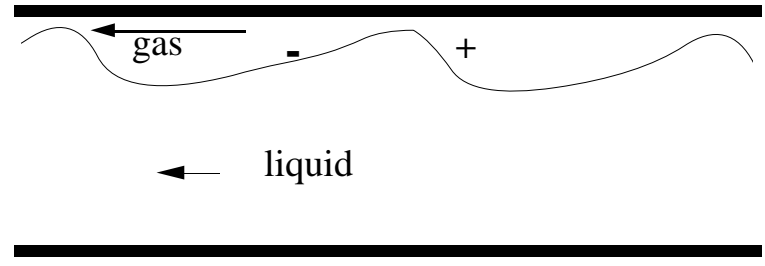

(b)

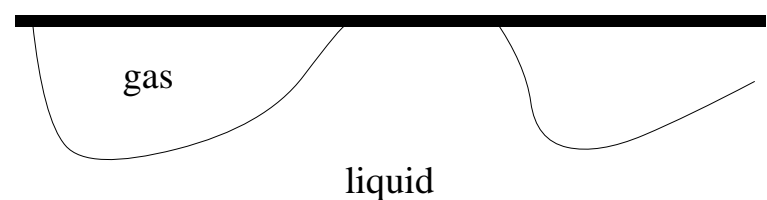

liquid

(c)

Figure 4. Slugging in gas-liquid flow. The high pressure at the front side steepens the wave and the low pressure at the back side smoothes it. If the amplitude of the wave is large enough it will touch the wall. Bernoulli effects can also suck the liquid to the wall when the gas velocity is large. Note that we have avoided drawing the ripples and small roll waves which are probably always superimposed on the gas-liquid interface. We focus on the macro-structures.

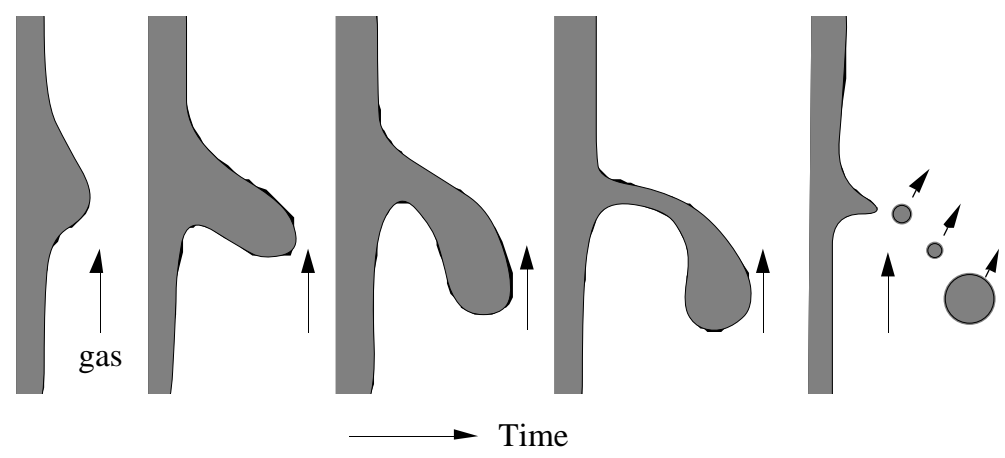

Figure 5. Breakdown of disturbance wave by "undercutting". (after Hewitt and Hall-Taylor [1970] ) 


\section{Concluding Remarks}

A criterion for the stabilization of annular flows based on the comparison of the effective viscosities of the fluids has been proposed here. Annular flow is stable when the fluid having the higher effective viscosity occupies the core region and lower viscosity fluid is in the annulus. This criterion can be expressed in terms of a two-phase Reynolds number involving the inertia of the gas phase in the tube and the viscosity of the liquid. The model is very simple and introduces another fundamental idea: stability of gas-liquid annular flow requires inertia of the gas. Comparison with existing flow maps for both horizontal and upward vertical annular flows show that indeed the proposed criterion is satisfied in almost the entire region of annular flow. The model is expected to hold only in forced convection flow. It is inappropriate to compare it with data for downflow because in this case gravity plays an important role in the stabilization of annular flow. Thus, the criterion may provide a method for determining conditions for stable pressure-driven annular flow for any pipe inclination. The criterion also appears to work in a variety of liquid-solid and gas-solid flows.

A mechanism for explaining the wave growth and initiation of slugs in gas-liquid flow, which is based on the action of a high stagnation pressure in the front of a initially small wave has been proposed. The same mechanism can explain the initiation of the "undercut" of wave crests and shatter of droplets observed in annular gas-liquid flows.

Acknowledgments - Dr. Bannwart's work at the University of Minnesota was supported by the Department of Energy of the State University of Campinas (UNICAMP) and FAPESP, Brazil. The work of Joseph and Liu was supported by the NSF-CTS, the DOE-Department of Basic Energy Sciences and the ARO, Mathematics.

\section{REFERENCES}

Bai, R., Kelkar, K., and Joseph, D.D. 1995 Direct simulation of interfacial waves in a high viscosity ratio and axisymmetric core annular flow. Report UMSI 95/107.. (accepted for publication in J.Fluid Mech. , 1996).

Duns, Jr., H., and Ros, N.C.J. 1963 Vertical flow of gas and liquid mixtures from boreholes. Proc. 6th World Petroleum Congress, Frankfurt, 451-465.

Fan, Z., Lusseyran, F. and Hanratty, T.J. 1993 Initiation of slugs in horizontal gas-liquid flows. AIChE J., 39, 1741-1753.

Feng, J., Huang, P. Y. and Joseph, D. D. 1995 Dynamic simulation of the motion of capsules in pipelines. J. Fluid Mech., 286, 201-227.

Griffith, P., and Wallis, G.B. 1961 Two-phase slug flow. J. Heat Trans., 83, 307-320.

Hewitt, G.F. and Hall-Taylor, N.S. 1970, Annular Two-Phase Flow. Pergamon Press, Oxford.

Iske, P.L., Sergeev, Y.A., Boersma, W.H. , Kurdyumov, V.N. and Moors, J.H.J. 1995 Particle phase boundary layer theory of solid concentration and velocity profiles in circulating fluidized bed risers. In Proc. 2nd Int. Conf. Multiphase Flow, Kyoto Japan, 33-37.

Joseph, D.D., Bai, R., Chen, K.P. and Renardy, Y.Y. 1996 Core annular flows. Report UMSI 96/31. (to appear in Ann. Rev. Fluid. Mech., 29, 1997).

Joseph, D.D. and Renardy, Y.Y. 1993 Fundamentals of Two-Fluid Dynamics, SpringerVerlag, New York.

Lane, W.R. 1951 Shatter of drops in streams of air. Ind. Eng. Chem., 43, 1312.

Mandhane, J.M., Gregory, G.A. and Aziz, K. 1974 A flow pattern map for gas-liquid flow in horizontal pipes. Int. J. Multiphase Flow, 1, 537-553. 
Merkle, C.L. and Deutsch, S. 1990 Drag reduction in boundary layers by gas injection. In Viscous Drag Reduction in Boundary Layers (Edited by Bushnell, D.M. and Hefner, T.M.). Prog. Astronaut. Aeronaut. 123, 351-412.

Persello, J., Magnin, A., Chang, J., Piau, J.M. and Cabane, B. 1994 Flow of colloidal aqueous silica dispersions. J. Rheology, 38, 1845-1870.

Sinclair, J.L. and Jackson, R. 1989 Gas-particle flow in a vertical pipe with particleparticle interactions. AIChE J., 35, 1473-1486.

Taitel, Y. and Dukler, A.E. 1976 A model for predicting flow regime transitions in horizontal and near horizontal gas-liquid flow. AIChE J., 22, 47-55.

Taitel, Y., Bornea, D. and Dukler, A.E. 1980 Modelling flow pattern transitions for steady upward gas-liquid flow in vertical tubes. AIChE J., 26, 345-354.

White, F.M. 1991 Viscous Fluid Flow. McGraw-Hill Book Co., New York, 438-439. 